\title{
Studying the Bessell Equation of Complex Order
}

Thair Y. Thanoon

\section{Omar Thaher shalal}

thairthanoon@uomosul.edu.iq

College of Computer Sciences and Mathematics

University of Mosul, Mosul, Iraq

Received on: 07/01/2019

Accepted on: 28/03/2019

\begin{abstract}
In this paper we derive Bessel equation of complex order $(n+i)$, after that generalized recurrence relations from Bessel equation of order (n) to Bessel equation of complex order $(n+i)$ and will satisfy that. We given illustrates example of different cases .
\end{abstract}

Keywords: Bessell equation, complex order.

\section{دراسة معادلة بيسل من الرتبة العقدية}

$$
\text { عدر ظاهر شلال }
$$

تاريخ قبول البحث: 2019103128

$$
\begin{aligned}
& \text { ثائر يونس ذنون } \\
& \text { قسم الرياضيات } \\
& \text { كلية علوم الحاسوب والرياضيات } \\
& \text { جامعة الموصل، الموصل، العراق }
\end{aligned}
$$

تاريخ استلام البحث: $2019 \backslash 01107$

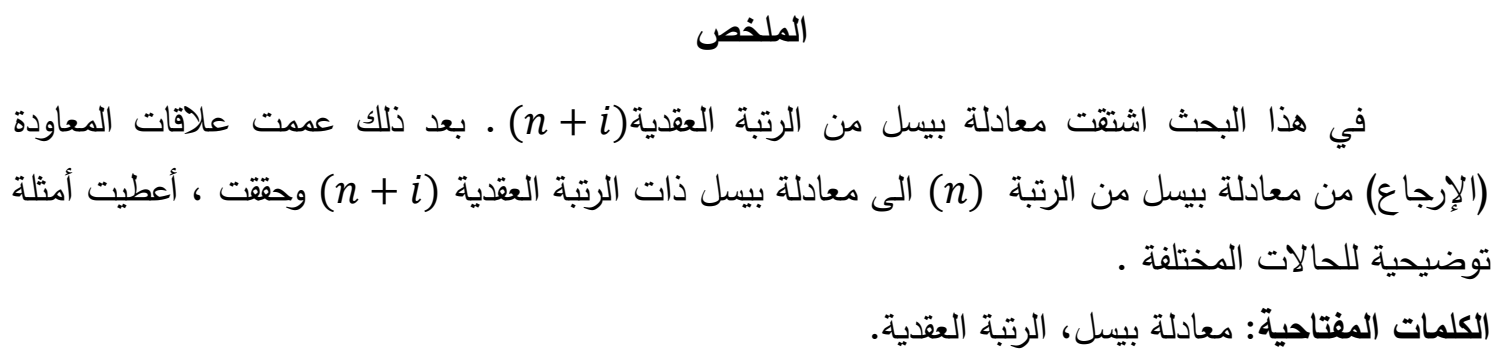

Introduction I المقدمة

المعادلة التفاضلية الخطية من الرتبة الثانية والمعاملات المتغيرة التالية

$x^{2} \frac{d^{2} y}{d x^{2}}+x \frac{d y}{d x}+\left(x^{2}-n^{2}\right) y=0 \quad, \quad n \geq 0$

تعرف (1.1) بمعادلة بيسل من الرتبة n (Bessels equation of order n) وتظهر هذه المعادلة

كثيراً في الرياضيات التطبيقية ومسائل الفيزياء والهندسة. وأي حل يحقق هذه المعادلة يسمى بدالة بيسل

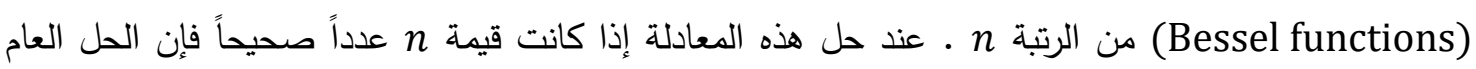

$y(x)=c_{1} J_{n}(x)+c_{2} y_{n}(x)$ 
إذ إن

$J_{n}(x)=\sum_{r=0}^{\infty} \frac{(-1)^{r}}{r !\lceil(n+r+1)}\left(\frac{x}{2}\right)^{n+2 r}$

$y_{n}(x)=\frac{1}{\sin n \pi}\left[J_{n}(x) \cos n \pi-J_{-n}(x)\right]$

أما اذا كانت n عدداً غير صحيحا فإن الحل العام يكون بالصيغة الآتية:

$y(x)=c_{1} J_{n}(x)+c_{2} J_{-n}(x)$

إذ إن (x) , J $J_{n}(x)$

$J_{n}(x)=\sum_{r=0}^{\infty} \frac{(-1)^{r}}{r ! \Gamma(n+r+1)}\left(\frac{x}{2}\right)^{n+2 r}$

ثوابت اختيارية وإن

$J_{-n}(x)=\sum_{r=0}^{\infty} \frac{(-1)^{r}}{r ! \Gamma(-n+r+1)}\left(\frac{x}{2}\right)^{-n+2 r}$

برنولي أول من أعطى مفهوم دالة بيسل في سنة (1732) إذ استخدم الدالة من الرتبة الصفرية حلاً لمسألة

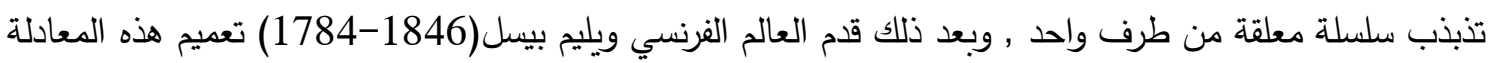

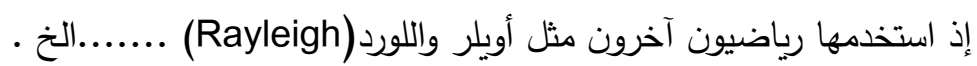

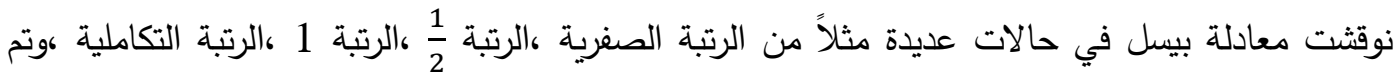

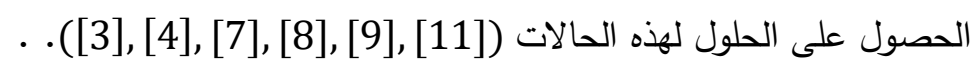

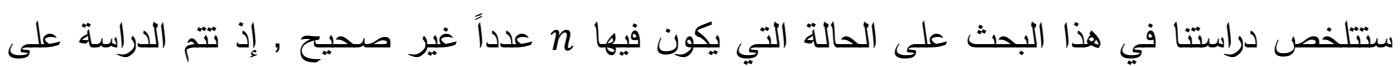

معادلة بيسل من الرتبة العقدية (n+i)

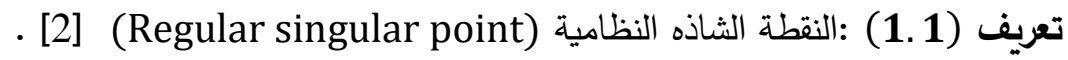
اذا كان لدينا المعادلة التقاضلية الآتية:

$\frac{d^{2} y}{d x^{2}}+p_{1}(x) \frac{d y}{d x}+p_{2}(x) y=0$

فأذا كانت كل من (x)

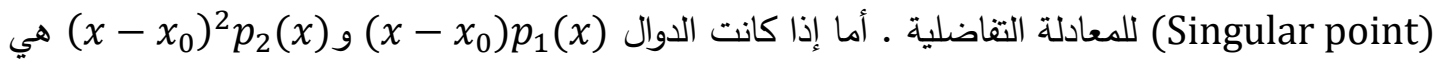

دوال تحليلية عند النقطة $x_{0}$ فإن هذه النقطة تسمى نقطة شاذة نظامية (Regular singular point) للدعادلة التفاضلية أما إذا كانت إحداهما أو كلاهما ليست دوالاً تحليلية عند النقطة $x_{0}$ فإن هذه النقطة تسمى نقطة

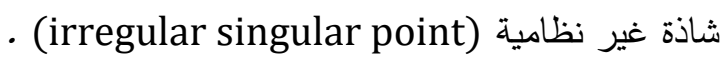
$\left\lceil(n)=\int_{0}^{\infty} x^{n-1} e^{-x} d x\right.$ تعريف (1.2): دالة كاما Gamma Function) (1. :تعرف بأنها

$\lceil(n+1)=n\lceil(n)$ التي تقترب عندما n> n م ـ وهنالك أيضاً صيغة أخرى كما وتعرف أيضاً بدالة المضروب أي أن 


$$
\lceil(n+1)=n !
$$

[8] (wronskian) تعريف (1.3): محدد رونسكيان

إذا كانت $w\left(y_{1}(x), y_{2}(x)\right)=\left|\begin{array}{ll}y_{1} & y_{2} \\ y_{1}^{\prime} & y_{2}^{\prime}\end{array}\right|=y_{1} y_{2}^{\prime}-y_{2} y_{1}^{\prime}$

إذا كانت $=0$ ف فإن الحلين معتمدان خطياً أما إذا كانت فإن الحلين مستقلان خطياً .

2- حل معادلة بيسل من الرتبة العقدية بطريقة متسلسلات القوى. لتكن المعادلة التفاضلية الخطية من الرتبة الثانية والمعاملات المتغيرة الآتية:

$x^{2} \frac{d^{2} y}{d x^{2}}+x \frac{d y}{d x}+\left(x^{2}-(n+i)^{2}\right) y=0 \quad, n \geq 0$

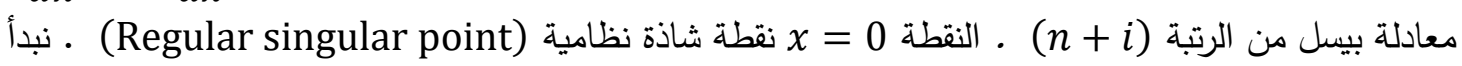

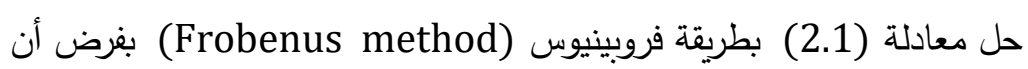
$y=x^{m}\left(a_{0}+a_{1} x+a_{2} x^{2}+a_{3} x^{3}+\ldots \ldots \ldots \ldots \ldots\right), \quad m>0$

$y=\sum_{r=0}^{\infty} a_{r} x^{m+r} \quad, \quad a_{r} \neq 0$

$\frac{d y}{d x}=\sum_{r=0}^{\infty} a_{r}(m+r) x^{m+r-1}$

$\frac{d^{2} y}{d x^{2}}=\sum_{r=0}^{\infty} a_{r}(m+r)(m+r-1) x^{m+r-2}$

نعوض المعادلة (2.2) والمعادلة (2.3) والمعادلة (2.4) في المعادلة (2.1) فنحصل على $x^{2} \sum_{r=0}^{\infty} a_{r}(m+r)(m+r-1) x^{m+r-2}+x \sum_{r=0}^{\infty} a_{r}(m+r) x^{m+r-1}$

$\sum_{r=0}^{\infty} a_{r}(m+r)(m+r-1) x^{m+r}+\sum_{r=0}^{\infty} a_{r}(m+r) x^{m+r}$

$$
+\left(x^{2}-(n+i)^{2}\right) \sum_{r=0}^{\infty} a_{r} x^{m+r}=0
$$$$
+\sum_{r=0}^{\infty} a_{r} x^{m+r+2}-(n+i)^{2} \sum_{r=0}^{\infty} a_{r} x^{m+r}=0
$$

$\sum_{r=0}^{\infty} a_{r}(m+r)(m+r-1) x^{m+r}+\sum_{r=0}^{\infty} a_{r}(m+r) x^{m+r}$

$+\sum_{r=2}^{\infty} a_{r-2} x^{m+r}-(n+i)^{2} \sum_{r=0}^{\infty} a_{r} x^{m+r}=0$
$m(m-1) a_{0} x^{m}+m(m+1) a_{1} x^{m+1}+m a_{0} x^{m}+(m+1) a_{1} x^{m+1}$
$-(n+i)^{2} a_{0} x^{m}-(n+i)^{2} a_{1} x^{m+1}$
$+\sum_{r=2}^{\infty}\left[\left[(m+r)(m+r-1)+(m+r)-(n+i)^{2}\right] a_{r}+a_{r-2}\right] x^{m+r}=0$

$\left[m(m-1)+m-(n+i)^{2}\right] a_{0}=0 \quad, \quad a_{0} \neq 0$

بمساواة معامل $m^{2}-(n+i)^{2}=0 \Rightarrow m^{2}=(n+i)^{2}$

$$
\begin{aligned}
& m=-(n+i) \quad \text { أما } \\
& \text { بمساواة معامل +1 بأ }
\end{aligned}
$$


$\left[m(m+1)+m+1-(n+i)^{2}\right] a_{1}=0$

$[2 m+1] a_{1}=0$

الحالة الأولى عندما

$$
[2 m+1] a_{1}=0
$$$$
\text { بما أن }
$$

$$
a_{1}=0
$$

$\left[(m+r)(m+r-1)+(m+r)-(n+i)^{2}\right] a_{r}+a_{r-2}=0$

$\left[m^{2}+2 m r+r^{2}-(n+i)^{2}\right] a_{r}=-a_{r-2}$

$\left[(n+i)^{2}+2(n+i) r+r^{2}-(n+i)^{2}\right] a_{r}=-a_{r-2}$

بما أن $m=n+i$ نحصل على

$r[2(n+i)+r] a_{r}=-a_{r-2}$

$a_{r}=\frac{-a_{r-2}}{r[2(n+i)+r]}$

$r \geq 2$

$a_{2}=\frac{-a_{0}}{2[2(n+i)+2]} \quad \Rightarrow a_{2}=\frac{-a_{0}}{2^{2}[(n+i)+1]}$

$a_{3}=\frac{-a_{1}}{3[2(n+i)+3]} \quad \because \quad a_{1}=0 \Rightarrow a_{3}=0$

$a_{4}=\frac{-a_{2}}{4[2(n+i)+4]} \Rightarrow a_{4}=\frac{-1}{4[2(n+i)+4]} \cdot \frac{-a_{0}}{2^{2}[(n+i)+1]}$

$a_{4}=\frac{a_{0}}{4[2(n+i)+4] 2^{2}[(n+i)+1]}$

$a_{5}=\frac{-a_{3}}{5[2(n+i)+5]} \quad \because \quad a_{3}=0 \Rightarrow a_{5}=0$

$a_{1}=a_{3}=a_{5}=a_{7}=\cdots=0$

إذن

وعليه

$a_{2 n-1}=0 \quad, \quad \mathrm{n}=1,2,3, \ldots \ldots \ldots . .$.

هذا يعني أن معاملات المعادلة (2.1) هي فقط المعاملات الزوجية. لذلك نعوض عن r في الصغغة التكرارية

$a_{2 k}=\frac{-a_{2 k-2}}{2 k[2(n+i)+2 k]} \quad, \quad k=1,2,3,4, \ldots$

$a_{2 k}=\frac{-a_{2 k-2}}{4 k[(n+i)+k]}$

$k=1 \quad \Rightarrow a_{2}=\frac{-a_{0}}{4[(n+i)+1]}$

$k=2 \Rightarrow a_{4}=\frac{-a_{2}}{4.2[(n+i)+2]} \quad \Rightarrow a_{4}=\frac{-1}{4.2[(n+i)+2]} \cdot \frac{-a_{0}}{4[(n+i)+1]}$

$\Rightarrow a_{4}=\frac{a_{0}}{4^{2} \cdot 2 \cdot 1[(n+i)+2][(n+i)+1]}$

$k=3 \Rightarrow a_{6}=\frac{-a_{4}}{4.3[(n+i)+3]}$

$\Rightarrow \quad a_{6}=\frac{-1}{4.3[(n+i)+3]} \cdot \frac{a_{0}}{4^{2} \cdot 2 \cdot 1[(n+i)+2][(n+i)+1]}$ 
$\Rightarrow a_{6}=\frac{-a_{0}}{4^{3} \cdot 3 \cdot 2 \cdot 1[(n+i)+3][(n+i)+2][(n+i)+1]}$

$a_{2 k}=\frac{(-1)^{k}}{4^{k} k ![(n+i)+k] \ldots \ldots \ldots \ldots \ldots .[(n+i)+1]}$

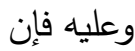

نعوض المعاملات في المعادلة (2.2) مع

$y=x^{n+i}\left(a_{0}-\frac{a_{0}}{4[(n+i)+1]} x^{2}+\frac{a_{0}}{4^{2} \cdot 2 \cdot 1[(n+i)+2][(n+i)+1]} x^{4}\right.$

$\left.-\frac{-a_{0}}{4^{3} \cdot 3 \cdot 2 \cdot 1[(n+i)+3][(n+i)+2][(n+i)+1]} x^{6}+\cdots\right)$

$y=a_{0} x^{n+i}\left(1-\frac{1}{4[(n+i)+1]} x^{2}+\frac{1}{4^{2} \cdot 2 \cdot 1[(n+i)+2][(n+i)+1]} x^{4}\right.$

$\left.-\frac{1}{4^{3} \cdot 3.2 .1[(n+i)+3][(n+i)+2][(n+i)+1]} x^{6}+\cdots\right)$.

إذا اخترنا قيمة الثابت a a

$a_{0}=\frac{1}{2^{n+i}(n+i) !}$

$$
\begin{aligned}
& y=\frac{1}{2^{n+i}(n+i) !} x^{n+i}\left(1-\frac{1}{4[(n+i)+1]} x^{2}+\frac{1}{4^{2} \cdot 2 \cdot 1[(n+i)+2][(n+i)+1]} x^{4}\right. \\
& \left.-\frac{1}{4^{3} \cdot 3 \cdot 2 \cdot 1[(n+i)+3][(n+i)+2][(n+i)+1]} x^{6}+\cdots\right) \\
& y=\left(\frac{x}{2}\right)^{n+i}\left[\frac{1}{(n+i) !}-\frac{1}{4[(n+i)+1] !} x^{2}-\frac{1}{4^{2} \cdot 2 \cdot 1[(n+i)+2] !} x^{4}\right. \\
& \left.-\frac{1}{4^{3} \cdot 3 \cdot 2 \cdot 1[(n+i)+3] !} x^{6}+\cdots\right]
\end{aligned}
$$

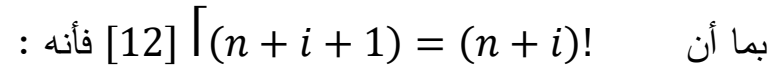$$
y=\left(\frac{x}{2}\right)^{n+i}\left[\frac{1}{\lceil(n+i+1)}-\frac{1}{4\lceil(n+i+2)} x^{2}+\frac{1}{4^{2} \cdot 2 \cdot 1\lceil(n+i+3)} x^{4}\right.
$$$$
\left.-\frac{1}{4^{3} \cdot 3 \cdot 2 \cdot 1\lceil(n+i+4)} x^{6}+\cdots\right]
$$$$
y=\left(\frac{x}{2}\right)^{n+i}\left[\frac{1}{\Gamma(n+i+1)}-\frac{1}{\lceil(n+i+2)}\left(\frac{x}{2}\right)^{2}+\frac{1}{2.1[(n+i+3)}\left(\frac{x}{2}\right)^{4}\right.
$$$$
\left.-\frac{1}{3.2 .1\lceil(n+i+4)}\left(\frac{x}{2}\right)^{6}+\cdots\right]
$$$$
y_{n+i}=J_{n+i}(x)=\sum_{r=0}^{\infty} \frac{(-1)^{r}}{r ! \Gamma(n+i+r+1)}\left(\frac{x}{2}\right)^{n+i+2 r}
$$

تسمى (x)

$a_{r}=\frac{-a_{r-2}}{r[r-2(n+i)]} \quad, \quad r \geq 2 \quad, \quad r \neq 2(n+i)$ 
وباستخدام الخطوات نفسها التي أجريناها للحصول على (x)

$J_{-(n+i)}(x)=\sum_{r=0}^{\infty} \frac{(-1)^{r}}{r ! \Gamma(-(n+i)+r+1)}\left(\frac{x}{2}\right)^{-(n+i)+2 r}$

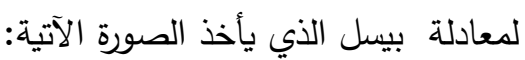

وتسمى هذه الدالة بدالة بيسل من النوع الأول والرتبة (i+i

$$
\begin{gathered}
y(x)=c_{1} J_{n+i}(x)+c_{2} J_{-(n+i)}(x) \\
x^{2} \frac{d^{2} y}{d x^{2}}+x \frac{d y}{d x}+\left(x^{2}-(3+i)^{2}\right) y=0
\end{gathered}
$$

n+ - n العقدية على الثكل الآتي:

الحل:

$y(x)=c_{1} J_{3+i}(x)+c_{2} J_{-3-i}(x)$

معادلة بيسل من الرتبةi + 3 والحل العام لهذا المعادلة هو

$$
\begin{aligned}
J_{3+i}(x)= & \sum_{r=0}^{\infty} \frac{(-1)^{r}}{r ! \Gamma(3+i+r+1)}\left(\frac{x}{2}\right)^{3+i+2 r} \\
J_{3+i}(x)= & \left(\frac{x}{2}\right)^{3+i}\left[\frac{1}{0 !\lceil(3+i+1)}-\frac{1}{1 ! \Gamma(3+i+2)}\left(\frac{x}{2}\right)^{2}+\frac{1}{2 ! \Gamma(3+i+3)}\left(\frac{x}{2}\right)^{4}\right. \\
& \left.\quad-\frac{1}{3 ! \Gamma(3+i+4)}\left(\frac{x}{2}\right)^{6}+\cdots\right] \\
J_{3+i}(x)= & \left(\frac{x}{2}\right)^{3+i}\left[\frac{1}{\lceil(4+i)}-\frac{1}{4\lceil(5+i)} x^{2}+\frac{1}{4^{2} \cdot 2 \cdot 1\lceil(6+i)} x^{4}\right.
\end{aligned}
$$$$
\left.-\frac{1}{4^{3} \cdot 3 \cdot 2 \cdot 1[(7+i)} x^{6}+\cdots\right]
$$$$
J_{3+i}(x)=\left(\frac{x}{2}\right)^{3+i}\left[\frac{1}{(3+i) !}-\frac{1}{4(4+i) !} x^{2}+\frac{1}{4^{2} \cdot 2 \cdot 1(5+i) !} x^{4}\right.
$$$$
\left.-\frac{1}{4^{3} \cdot 3 \cdot 2 \cdot 1(6+i) !} x^{6}+\cdots\right]
$$$$
J_{3+i}(x)=\left(\frac{x}{2}\right)^{3+i}\left[\frac{1}{1.55+4.98 i}-\frac{1}{4(1.22+21.47 i)} x^{2}+\frac{1}{32(-15.37+108.57 i)} x^{4}\right.
$$$$
\left.-\frac{1}{384(-200.79+636.05 i)} x^{6}+\cdots\right]
$$$$
J_{3+i}(x)=\left(\frac{x}{2}\right)^{3+i}\left[0.0569-0.18306 i-(0.00065-0.0116 i) x^{2}\right.
$$$$
\left.-(0.00003+0.0002 i) x^{4}+(0.000001+0.000003 i) x^{6}+\cdots\right]
$$$$
J_{-3-i}(x)=\sum_{r=0}^{\infty} \frac{(-1)^{r}}{r ![(-3-i+r+1)}\left(\frac{x}{2}\right)^{-3-i+2 r}
$$ 


$$
\begin{aligned}
& J_{-3-i}(x)=\left(\frac{x}{2}\right)^{-3-i}\left[\frac{1}{0 !\lceil(-3-i+1)}-\frac{1}{1 !\lceil(-3-i+2)}\left(\frac{x}{2}\right)^{2}\right. \\
& \left.+\frac{1}{2 !\lceil(-3-i+3)}\left(\frac{x}{2}\right)^{4}-\frac{1}{3 !\lceil(-3-i+4)}\left(\frac{x}{2}\right)^{6}+\cdots\right] \\
& J_{-3-i}(x)=\left(\frac{x}{2}\right)^{-3-i}\left[\frac{1}{\lceil(-2-i)}-\frac{1}{4\lceil(-1-i)} x^{2}+\frac{1}{4^{2} \cdot 2.1\lceil(-i)} x^{4}\right. \\
& \left.-\frac{1}{4^{3} \cdot 3 \cdot 2 \cdot 1\lceil(1-i)} x^{6}+\cdots\right] \\
& J_{-3-i}(x)=\left(\frac{x}{2}\right)^{-3-i}\left[\frac{1}{(-3-i) !}-\frac{1}{4(-2-i) !} x^{2}+\frac{1}{4^{2} \cdot 2 \cdot 1(-1-i) !} x^{4}\right. \\
& \left.-\frac{1}{4^{3} \cdot 3 \cdot 2 \cdot 1(-i) !} x^{6}+\cdots\right] \\
& J_{-3-i}(x)=\left(\frac{x}{2}\right)^{-3-i}\left[\frac{10}{1.339+0.963 i}-\frac{1}{2(-0.343-0.653 i)} x^{2}\right. \\
& \left.+\frac{1}{32(-0.155+0.498 i)} x^{4}-\frac{1}{384(0.498+0.155 i)} x^{6}+\cdots\right] \\
& J_{-3-i}(x)=\left(\frac{x}{2}\right)^{-3-i}\left[\begin{array}{c}
4.9224-3.5401 i+(0.3152-0.6001 i) x^{2} \\
-(0.0178+0.0572 i) x^{4}-(0.0047-0.0014 i) x^{6}+\cdots
\end{array}\right]
\end{aligned}
$$

قضية(2.1):

إذا كانت (x)

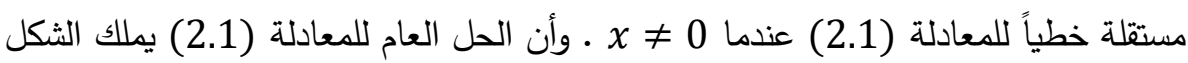
$y(x)=c_{1} J_{n+i}(x)+c_{2} J_{-(n+i)}(x)$

إذ إن c و $c_{1}$ هي ثوابت أخيارية .

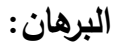

لإثبات أن دوال بيسل ${ }^{\prime}$

$W\left(J_{n+i}(x), J_{-(n+i)}(x)\right)=\left|\begin{array}{ll}J_{n+i}(x) & J_{-(n+i)}(x) \\ J_{n+i}^{\prime}(x) & J_{-(n+i)}^{\prime}(x)\end{array}\right|$

• لا يساوي صفر (wronskian)

بما أن (2.1) $x^{2} J_{n+i}^{\prime \prime}(x)+x J_{n+i}^{\prime}(x)+\left(x^{2}-(n+i)^{2}\right) J_{n+i}(x)=0$

$x^{2} J_{-(n+i)}^{\prime \prime}(x)+x J_{-(n+i)}^{\prime}(x)+\left(x^{2}-(n+i)^{2}\right) J_{-(n+i)}(x)=0$

نضرب هاتين المعادلتين بواسطة ${ }^{\prime} J_{n+i}(x), J_{-(n+i)}(x)$ على التوالي وطرحهما من بعضهما وقسمة الناتج

$$
\begin{gathered}
x\left(J_{n+i}(x) J^{\prime \prime}-(n+i)\right. \\
-J_{-(n+i)}(x) J_{n+i}^{\prime}(x)=0
\end{gathered}
$$




$$
\begin{aligned}
& \frac{d}{d x}\left(x J_{n+i}(x) J_{-(n+i)}^{\prime}(x)-J_{-(n+i)}(x) J_{n+i}^{\prime}(x)\right. \\
& =\frac{d}{d x}\left(x W\left(J_{n+i}(x), J_{-(n+i)}(x)\right)=0\right. \\
& W\left(J_{n+i}(x), J_{-(n+i)}(x)\right)=\frac{c}{x} \\
& W\left(J_{n+i}(x), J_{-(n+i)}(x)\right) \neq 0
\end{aligned}
$$

إذن دوال بيسل (x) مبرهنة(2.2): إذا كانت

$$
\begin{aligned}
& J_{n+i}(x)=\sum_{r=0}^{\infty} \frac{(-1)^{r}}{r !\lceil(n+i+r+1)}\left(\frac{x}{2}\right)^{n+i+2 r}, n>-1 \\
& \lim _{x \rightarrow 0} \frac{J_{n+i}(x)}{x^{n+i}}=\frac{1}{2^{n+i}\lceil(n+i+1)}
\end{aligned}
$$

(2.8) (البرهان: من المعادلة (2.)

$$
\begin{gathered}
J_{n+i}(x)=\left(\frac{x}{2}\right)^{n+i}\left[\frac{1}{[(n+i+1)}-\frac{1}{4\lceil(n+i+2)} x^{2}+\frac{1}{4^{2} .2 .1\lceil(n+i+3)} x^{4}\right. \\
\left.-\frac{1}{4^{3} \cdot 3.2 .1\lceil(n+i+4)} x^{6}+\cdots\right]
\end{gathered}
$$

$$
\begin{gathered}
\frac{J_{n+i}(x)}{x^{n+i}}=\frac{1}{2^{n+i} \Gamma_{(n+i+1)}}\left[1-\frac{1}{4(n+i+1)} x^{2}+\frac{1}{4^{2} \cdot 2 \cdot 1(n+i+2)(n+i+1)} x^{4}\right. \\
\left.-\frac{1}{4^{3} \cdot 3 \cdot 2 \cdot 1(n+i+3)(n+i+2)(n+i+1)} x^{6}+\cdots\right]
\end{gathered}
$$

$$
\begin{aligned}
\lim _{x \rightarrow 0} \frac{J_{n+i}(x)}{x^{n+i}} & =\lim _{x \rightarrow 0} \frac{1}{2^{n+i} \Gamma_{(n+i+1)}}\left[1-\frac{1}{4(n+i+1)} x^{2}\right. \\
& +\frac{1}{4^{2} \cdot 2 \cdot 1(n+i+2)(n+i+1)} x^{4} \\
& \left.-\frac{1}{4^{3} \cdot 3 \cdot 2 \cdot 1(n+i+3)(n+i+2)(n+i+1)} x^{6}+\cdots\right]
\end{aligned}
$$

$\lim _{x \rightarrow 0} \frac{J_{n+i}(x)}{x^{n+i}}=\frac{1}{2^{n+i} \Gamma_{(n+i+1)}}$

$$
J_{-(n+i)}(x)=(-1)^{n+i} J_{n+i}(x)
$$

$$
\begin{aligned}
& J_{-(n+i)}(x)=\sum_{r=0}^{\infty} \frac{(-1)^{r}}{r ! \Gamma(-(n+i)+r+1)}\left(\frac{x}{2}\right)^{-(n+i)+2 r} \\
& =\sum_{r=0}^{(n+i)-1} \frac{(-1)^{r}\left(\frac{x}{2}\right)^{-(n+i)+2 r}}{r ! \Gamma(-(n+i)+r+1)}+\sum_{r=n+i}^{\infty} \frac{(-1)^{r} \quad\left(\frac{x}{2}\right)^{-(n+i)+2 r}}{r ! \Gamma(-(n+i)+r+1)} \\
& =0+\sum_{r=n+i}^{\infty} \frac{(-1)^{r}\left(\frac{x}{2}\right)^{-(n+i)+2 r}}{r ![(-(n+i)+r+1)}
\end{aligned}
$$




$$
\begin{gathered}
\text { الآن نضع بدلاً عن } J_{-(n+i)}(x)=\sum_{k=0}^{\infty} \frac{(-1)^{(n+i)+k}\left(\frac{x}{2}\right)^{(n+i)+2 k}}{((n+i)+k) !(k+1)}=(-1)^{n+i} \sum_{k=0}^{\infty} \frac{(-1)^{k}\left(\frac{x}{2}\right)^{n+i+2 k}}{k ! \Gamma(n+i+k+1)} \\
J_{-(n+i)}(x)=(-1)^{n+i} J_{n+i}(x)
\end{gathered}
$$

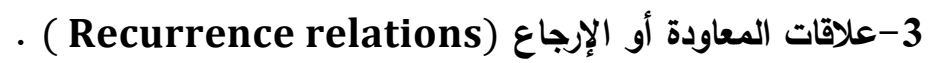

ذكرت علاقات المعاودة أو الإرجاع في [3] .في حالة n عدد صحيح .سنتاول هذه العلاقات في الحالة

$$
\text { العقدية (n+i) }
$$

قضية(3.1) : (3.1) إذا كانت

$$
J_{n+i}=\sum_{r=0}^{\infty} \frac{(-1)^{r}}{r !\lceil(n+i+r+1)}\left(\frac{x}{2}\right)^{n+i+2 r}
$$$$
\text { 1. } x J_{n+i}=(n+i) J_{n+i}-x J_{n+1+i}
$$$$
\text { 2. } x J_{n+i}=-(n+i) J_{n+i}+x J_{n-1+i}
$$

$$
x J_{n+i}=(n+i) J_{n+i}-x J_{n+1+i}
$$

$$
\begin{aligned}
& J_{n+i}=\sum_{r=0}^{\infty} \frac{(-1)^{r}}{r !\lceil(n+i+r+1)}\left(\frac{x}{2}\right)^{n+i+2 r} \\
& J_{n+i}=\sum_{r=0}^{\infty} \frac{(-1)^{r}(n+i+2 r)}{r !\lceil(n+i+r+1)}\left(\frac{x}{2}\right)^{n+i+2 r-1} \frac{1}{2}
\end{aligned}
$$$$
=(n+i) \sum_{r=0}^{\infty} \frac{(-1)^{r}}{r !\lceil(n+i+r+1)}\left(\frac{x}{2}\right)^{n+i+2 r-1} \frac{1}{2}
$$

$$
\begin{aligned}
& +\sum_{r=0}^{\infty} \frac{(-1)^{r} 2 r}{r !\lceil(n+i+r+1)}\left(\frac{x}{2}\right)^{n+i+2 r-1} \frac{1}{2} \\
& x j_{n+i}=x(n+i) \sum_{r=0}^{\infty} \frac{(-1)^{r}}{r !\lceil(n+i+r+1)}\left(\frac{x}{2}\right)^{n+i+2 r-1} \quad \frac{1}{2} \\
& +x \sum_{r=1}^{\infty} \frac{(-1)^{r} 2}{(r-1) !\lceil(n+i+r+1)}\left(\frac{x}{2}\right)^{n+i+2 r-1} \quad \frac{1}{2}
\end{aligned}
$$

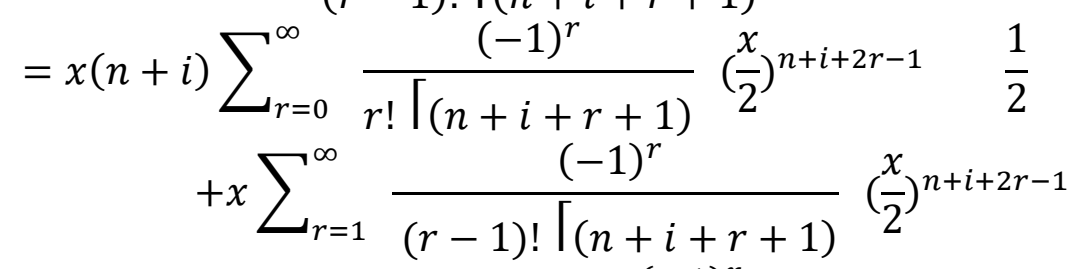$$
=(n+i) J_{n+i}+x \sum_{r=1}^{\infty} \frac{(-1)^{r}}{(r-1) !\lceil(n+i+r+1)}\left(\frac{x}{2}\right)^{n+i+2 r-1}
$$ 


$$
\begin{gathered}
x J_{n+i}=(n+i) J_{n+i}+x \sum_{s=0}^{\infty} \frac{(-1)^{s+1}}{s !\lceil(n+i+s+2)}\left(\frac{x}{2}\right)^{n+i+2 s+1} \\
x J_{n+i}^{\infty}=(n+i) J_{n+i}-x \sum_{s=0}^{\infty} \frac{(-1)^{s}}{s !\lceil(n+i+s+2)}\left(\frac{x}{2}\right)^{n+i+2 s+1} \\
x J_{n+i}^{\prime}=(n+i) J_{n+i}-x J_{n+1+i}
\end{gathered}
$$

$$
x J_{n+i}=-(n+i) J_{n+i}+x J_{n-1+i}
$$

$$
\begin{aligned}
& J_{n+i}=\sum_{r=0}^{\infty} \frac{(-1)^{r}}{r !\lceil(n+i+r+1)}\left(\frac{x}{2}\right)^{n+i+2 r} \\
& J_{n+i}=\sum_{r=0}^{\infty} \frac{(-1)^{r}(n+i+2 r)}{r ! \Gamma(n+i+r+1)}\left(\frac{x}{2}\right)^{n+i+2 r-1} \frac{1}{2}
\end{aligned}
$$$$
=\sum_{r=0}^{\infty} \frac{(-1)^{r}[2(n+i)+2 r-(n+i)]}{r !\lceil(n+i+r+1)}\left(\frac{x}{2}\right)^{n+i+2 r-1} \frac{1}{2}
$$$$
x j_{n+i}=\sum_{r=0}^{\infty} \frac{(-1)^{r}[2(n+i)+2 r-(n+i)]}{r ![(n+i+r+1)}\left(\frac{x}{2}\right)^{n+i+2 r}
$$

$$
\begin{aligned}
& =\sum_{r=0}^{\infty} \frac{(-1)^{r}[2(n+i)+2 r]}{r ![(n+i+r+1)}\left(\frac{x}{2}\right)^{n+i+2 r} \\
& -(n+i) \sum_{r=0}^{\infty} \frac{(-1)^{r}}{r !\lceil(n+i+r+1)}\left(\frac{x}{2}\right)^{n+i+2 r} \\
& x J_{n+i}=\sum_{r=0}^{\infty} \frac{(-1)^{r} 2[(n+i)+r]}{r ![(n+i+r+1)}\left(\frac{x}{2}\right)^{n+i+2 r}-(n+i) J_{n+i} \\
& x J_{n+i}=\sum_{r=0}^{\infty} \frac{(-1)^{r} 2[(n+i)+r]}{r !(n+i+r)[(n+i+r)}\left(\frac{x}{2}\right)^{n+i+2 r}-(n+i) J_{n+i} \\
& x J_{n+i}=\sum_{r=0}^{\infty} \frac{(-1)^{r} 2}{r ![(n+i+r)}\left(\frac{x}{2}\right)^{n+i+2 r}-(n+i) J_{n+i} \\
& x J_{n+i}=x \sum_{r=0}^{\infty} \frac{(-1)^{r}}{r ![(n+i+r)}\left(\frac{x}{2}\right)^{n+i+2 r-1}-(n+i) J_{n+i} \\
& x J_{n+i}=x \sum_{r=0}^{\infty} \frac{(-1)^{r}}{r ![(n+i+r)}\left(\frac{x}{2}\right)^{n-1+i+2 r}-(n+i) J_{n+i} \\
& x J_{n+i}=x J_{n-1+i}-(n+i) J_{n+i}
\end{aligned}
$$$$
J_{n+i}=\sum_{r=0}^{\infty} \frac{(-1)^{r}}{r !\lceil(n+i+r+1)}\left(\frac{x}{2}\right)^{n+i+2 r}
$$

3. $2 J_{n+i}=J_{n-1+i}-J_{n+1+i}$ 
4. $2(n+i) J_{n+i}=x\left(J_{n-1+i}+J_{n+1+i}\right)$

$2 J_{n+i}^{\prime}=J_{n-1+i}-J_{n+1+i}$

$x J_{n+i}=(n+i) J_{n+i}-x J_{n+1+i}$

$x J_{n+i}=-(n+i) J_{n+i}+x J_{n-1+i}$

$2 x \grave{n+i}_{n}=-x J_{n+1+i}+x J_{n-1+i}$

$2 J_{n+i}=-J_{n+1+i}+J_{n-1+i}$

$2 J_{n+i}=J_{n-1+i}-J_{n+1+i}$

$2(n+i) J_{n+i}=x\left(J_{n-1+i}+J_{n+1+i}\right)$

$x J_{n+i}=(n+i) J_{n+i}-x J_{n+1+i}$

$x J_{n+i}=-(n+i) J_{n+i}+x J_{n-1+i}$

$-(n+i) J_{n+i}+x J_{n-1+i}=(n+i) J_{n+i}-x J_{n+1+i}$

$2(n+i) J_{n+i}-x J_{n+1+i}-x J_{n-1+i}=0$

$2(n+i) J_{n+i}=x J_{n+1+i}+x J_{n-1+i}$

$2(n+i) J_{n+i}=x\left(J_{n+1+i}+J_{n-1+i}\right)$

$J_{n+i}=\sum_{r=0}^{\infty} \frac{(-1)^{r}}{r !\lceil(n+i+r+1)}\left(\frac{x}{2}\right)^{n+i+2 r}$

5. $\frac{d}{d x}\left(x^{-(n+i)} J_{n+i}\right)=-x^{-(n+i)} J_{n+1+i}$

6. $\frac{d}{d x}\left(x^{(n+i)} J_{n+i}\right)=x^{(n+i)} J_{n-1+i}$

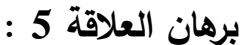

$\frac{d}{d x}\left(x^{-(n+i)} J_{n+i}\right)=-x^{-(n+i)} J_{n+1+i}$

$J_{n+i}=\sum_{r=0}^{\infty} \frac{(-1)^{r}}{r !\lceil(n+i+r+1)}\left(\frac{x}{2}\right)^{n+i+2 r}$

$x^{-(n+i)} J_{n+i}=\sum_{r=0}^{\infty} \frac{(-1)^{r} x^{2 r}}{r ! 2^{n+i+2 r}\lceil(n+i+r+1)}$
برهان العلاقة 3 : - (

من القضية(3.1) نحصل على (3.1) - ملى

بجمع (3.1) و(3.2) نحصل على

بالقسمة على x

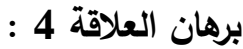

من القضية(3.1) نحصل على

قضية(3.3):إذا كانت

فإن 


$$
\begin{aligned}
& \frac{d}{d x}\left(x^{-(n+i)} J_{n+i}\right)=\sum_{r=0}^{\infty} \frac{(-1)^{r} 2 r x^{2 r-1}}{r ! 2^{n+i+2 r}\lceil(n+i+r+1)} \\
& =\sum_{r=1}^{\infty} \frac{(-1)^{r} 2 x^{2 r-1}}{(r-1) ! 2^{n+i+2 r}\lceil(n+i+r+1)} \\
& \text { r } \\
& =\sum_{k=0}^{\infty} \frac{(-1)^{k+1} 2 x^{2 k+1}}{k ! 2^{n+i+2 k+2}\lceil(n+i+k+2)} \\
& =\sum_{k=0}^{\infty} \frac{(-1)^{k+1} 2 x^{n+i+2 k+1} x^{-(n+i)}}{k ! 2^{n+i+2 k+1} 2\lceil(n+i+k+2)} \\
& =x^{-(n+i)} \sum_{k=0}^{\infty} \frac{(-1)^{k}(-1) x^{n+i+2 k+1}}{k ! 2^{n+i+2 k+1}\lceil(n+i+k+2)}
\end{aligned}
$$

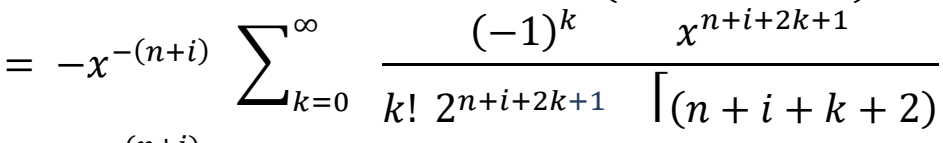

$$
\begin{aligned}
& =-x^{-(n+i)} J_{n+1+i}
\end{aligned}
$$

برهان العلاقة 6 :

$\frac{d}{d x}\left(x^{(n+i)} J_{n+i}\right)=x^{(n+i)} J_{n-1+i}$

$$
\begin{aligned}
& J_{n+i}=\sum_{r=0}^{\infty} \frac{(-1)^{r}}{r !\lceil(n+i+r+1)}\left(\frac{x}{2}\right)^{n+i+2 r} \\
& x^{n+i} \quad J_{n+i}=\sum_{r=0}^{\infty} \frac{(-1)^{r} x^{2(n+i)+2 r}}{r ! 2^{n+i+2 r}\lceil(n+i+r+1)} \\
& \frac{d}{d x}\left[x^{n+i} J_{n+i}\right]=\sum_{r=0}^{\infty} \frac{(-1)^{r}(2(n+i)+2 r) x^{2(n+i)+2 r-1}}{r ! 2^{n+i+2 r}\lceil(n+i+r+1)}
\end{aligned}
$$$$
\frac{d}{d x}\left[x^{n+i} J_{n+i}\right]=x^{n+i} \sum_{r=0}^{\infty} \frac{(-1)^{r} 2(n+i) x^{n+i+2 r-1}}{r ! 2^{n+i+2 r}\lceil(n+i+r+1)}
$$

$$
\begin{aligned}
\frac{d}{d x}\left[x^{n+i} J_{n+i}\right]= & x^{n+i} \sum_{r=0}^{\infty} \frac{(-1)^{r}(n+i) x^{n+i+2 r-1}}{r !} \sum_{r=0}^{\infty} \frac{(-1)^{r} 2 r x^{n+i+2 r-1}}{r ! 2^{n+i+2 r}\lceil(n+i+r+1)} \\
& +x^{n+i} \sum_{r=0}^{\infty} \frac{(-1)^{r} r x^{n+i+2 r-1}}{r ! 2^{n+i+2 r-1}\lceil(n+i+r+1)} \\
= & x^{n+i} \sum_{r=0}^{\infty} \frac{(-1)^{r}\left(\frac{x}{2}\right)^{n+i+2 r-1}(n+i+r)}{r !\lceil(n+i+r+1)} \\
= & x^{n+i} \sum_{r=0}^{\infty} \frac{(-1)^{r}\left(\frac{x}{2}\right)^{n+i+2 r-1}(n+i+r)}{r !(n+i+r)\lceil(n+i+r)}
\end{aligned}
$$




$$
\begin{aligned}
& =x^{n+i} \sum_{r=0}^{\infty} \frac{(-1)^{r}\left(\frac{x}{2}\right)^{n+i+2 r-1}}{r !\lceil(n+i+r)} \\
\frac{d}{d x}\left(x^{(n+i)} J_{n+i}\right) & =x^{(n+i)} J_{n-1+i}
\end{aligned}
$$

أمثلة توضيحية كتطبيق للعلاقات السابقة .

مثال(1): اكتب دالة بيسل $J_{5+i} J_{1+i}$ بدلالة كل من دوال بيسل $J_{2+i}$

$$
\begin{aligned}
& 2(n+i) J_{n+i}=x\left(J_{n-1+i}+J_{n+1+i}\right) \\
& 2(4+i) J_{4+i}=x\left(J_{3+i}+J_{5+i}\right) \\
& \left(\frac{8+2 i}{x}\right) J_{4+i}=\left(J_{3+i}+J_{5+i}\right) \\
& J_{5+i}=\left(\frac{8+2 i}{x}\right) J_{4+i}-J_{3+i} \\
& 2(3+i) J_{3+i}=x\left(J_{2+i}+J_{4+i}\right) \\
& \left(\frac{6+2 i}{x}\right) J_{3+i}=\left(J_{2+i}+J_{4+i}\right) \\
& J_{4+i}=\left(\frac{6+2 i}{x}\right) J_{3+i}-J_{2+i} \\
& 2(2+i) J_{2+i}=x\left(J_{1+i}+J_{3+i}\right) \\
& \left(\frac{4+2 i}{x}\right) J_{2+i}=\left(J_{1+i}+J_{3+i}\right) \\
& J_{3+i}=\left(\frac{4+2 i}{x}\right) J_{2+i}-J_{1+i}
\end{aligned}
$$

الحل: العلاقة الرابعة من نتيجة (3.2)

نعوض المعادلة (3.7) والمعادلة (3.8) في المعادلة (3.6) فنحصل على $J_{5+i}=\left(\frac{8+2 i}{x}\right)\left(\left(\frac{6+2 i}{x}\right)\left[\left(\frac{4+2 i}{x}\right) J_{2+i}-J_{1+i}\right]-J_{2+i}\right)-\left[\left(\frac{4+2 i}{x}\right) J_{2+i}-J_{1+i}\right]$ $J_{5+i}=\frac{44+28 i}{x}\left(\frac{4+2 i}{x} J_{2+i}-J_{1+i}\right)-\frac{8+2 i}{x} J_{2+i}-\frac{4+2 i}{x} J_{2+i}+J_{1+i}$ $J_{5+i}=\frac{120+200 i}{x^{3}} J_{2+i}-\frac{44+28 i}{x^{2}} J_{1+i}-\frac{8+2 i}{x} J_{2+i}-\frac{4+2 i}{x} J_{2+i}+J_{1+i}$ $J_{5+i}=\frac{120+200 i}{x^{3}} J_{2+i}-\frac{44+28 i}{x^{2}} J_{1+i}-\frac{12+4 i}{x} J_{2+i}+J_{1+i}$ $J_{5+i}=\left[1-\frac{44+28 i}{x^{2}}\right] J_{1+i}+\left[\frac{120+200 i}{x^{3}}-\frac{12+4 i}{x}\right] J_{2+i}$

$$
\frac{d}{d x}\left[J_{n+i}^{2}(x)+J_{n+1+i}^{2}(x)\right]=2\left[\frac{n+i}{x} J_{n+i}^{2}(x)-\frac{(n+1+i)}{x} J_{n+1+i}^{2}(x)\right]
$$
البرهان : من القضية (3.1) نحصل على

$$
\begin{aligned}
& x J_{n+i}(x)=(n+i) J_{n+i}(x)-x J_{n+1+i}(x) \\
& x J_{n+i}^{\prime}(x)=-(n+i) J_{n+i}(x)+x J_{n-1+i}(x)
\end{aligned}
$$


نضع الصيغة(3.10) بدلاً عن كل n+13 (x)

$x J_{n+1+i}(x)=-(n+1+i) J_{n+1+i}(x)+x J_{n+i}(x)$

$\frac{d}{d x}\left[J_{n+i}^{2}(x)+J_{n+1+i}^{2}(x)\right]=2 J_{n+i}(x) J_{n+i}^{\prime}(x)+2 J_{n+1+i}(x) J_{n+1+i}^{\prime}(x)$

قيم (x)

$$
\begin{aligned}
\frac{d}{d x}\left[J_{n+i}^{2}(x)+J_{n+1+i}^{2}(x)\right]=2 J_{n+i}(x) & {\left[\frac{n+i}{x} J_{n+i}(x)-J_{n+1+i}(x)\right] } \\
+ & 2 J_{n+1+i}(x)\left[\frac{-(n+1+i)}{x} J_{n+1+i}(x)+J_{n+i}(x)\right]
\end{aligned}
$$

$\frac{d}{d x}\left[J_{n+i}^{2}(x)+J_{n+1+i}^{2}(x)\right]=\frac{2}{x} J_{n+i}(x)\left[(n+i) J_{n+i}(x)-x J_{n+1+i}(x)\right]$

$$
\begin{array}{r}
+\frac{2}{x} J_{n+1+i}(x)\left[-(n+1+i) J_{n+1+i}(x)+x J_{n+i}(x)\right] \\
=2 \frac{n+i}{x} J_{n+i}^{2}(x)-2 J_{n+i}(x) J_{n+1+i}(x)-2 \frac{(n+1+i)}{x} J_{n+1+i}^{2}(x)
\end{array}
$$$$
+2 J_{n+1+i}(x) J_{n+i}(x)
$$$$
=2\left[\frac{n+i}{x} J_{n+i}^{2}(x)-\frac{(n+1+i)}{x} J_{n+1+i}^{2}(x)\right]
$$

مثال(3): إذا كانت

$\int_{0}^{x} x^{-(n+i)} J_{n+1+i}(x) d x=\frac{1}{2^{n+i}\lceil(n+i+1)}-x^{-(n+i)} J_{n+i}(x)$

البرهان : العلاقة الخامسة من قضية (3.3) نحصل على

$\frac{d}{d x}\left(x^{-(n+i)} J_{n+i}\right)=-x^{-(n+i)} J_{n+1+i}$

$\int_{0}^{x} x^{-(n+i)} J_{n+1+i}(x) d x=-\left[x^{-(n+i)} J_{n+i}(x)\right]_{0}^{x}$

$$
=-x^{-(n+i)} J_{n+i}+\lim _{x \rightarrow 0} \frac{J_{n+i}(x)}{x^{n+i}}
$$$$
=-x^{-(n+i)} J_{n+i}+\frac{1}{2^{n+i}\lceil(n+i+1)}
$$$$
=\frac{1}{2^{n+i} \Gamma_{(n+i+1)}}-x^{-(n+i)} J_{n+i}(x)
$$ 


\section{المصادر}

[1] Artin, E. The gamma function. Courier Dover Publications , (2015).

[2] Bowm[2] Bowman, F. Introduction to Bessel functions. Courier Corporation, Springer, (2012).

[3] Dass H. K. Er. Rajnish Verma, "Higher Engineering Mathematics", First Edition, Springer, (2011).

[4] Fedoryuk, M. V. Asymptotic analysis: linear ordinary differential equations. Springer Science \& Business Media,(2012).

[5] Korenev B. G. "Bessel Functions And Their Applications"CRC Press, Springer, (2003).

[6] Kreyszig E. "Advanced Engineering Mathematics", Wiley, Springer , (2013) .

[7] Luke, Y. L. Integrals of Bessel functions. Courier Corporation, (2014) .

[8] Markel E. G., "Bessel Functions and Equations of Mathematical Physics", Supervisor, Judith Rivas Ulloa, Leioa, 25 June (2015).

[9] Mclachlan N. W. "Bessel Functions For Engineers", $2^{\text {nd }}$ Edition, Oxford University, Press, London, (1955).

[10] Okrasinski W., L. Płociniczak, "A Note on Fractional Bessel Equation and Its Asymptotics", Fract. Calc. Appl. Anal., 16 (2013).

[11] Robin W., "Ladder-operator Factorization and the Bessel Differential Equations", Math. Forum, 9 (2014).

[12] Thukral A. K. "Factorials Of Real Negative And Imaginary Numbers-A new Perspetive", Published (2014). 